\title{
On the indexing and reciprocal space of icosahedral quasicrystal
}

\author{
Alok Singh and S. Ranganathan \\ Centre for Advanced Study, Department of Metallurgy, Indian Institute of Science, \\ Bangalore, 560 012, India
}

(Received 13 April 1998; accepted 2 August 1999)

\begin{abstract}
Important features of the icosahedral reciprocal space have been brought out. All reciprocal vectors up to sixth generation (by addition of icosahedral vectors) have been considered. Some more relationships for indexing the icosahedral phase are derived, and it is shown that the zone law using Cahn indices is also analogous to that valid for crystals. All important vectors, i.e., up to fourth generation and sixth generation, have been identified. Poles of all these vectors have been determined and shown to be one of the zone axes formed by these vectors. The types of indices that the planes and axes will have in three-dimensional and six-dimensional coordinates is discussed.
\end{abstract}

\section{INTRODUCTION}

Excitement was created by the discovery of the icosahedral phase by Shechtman, Blech, Gratias, and Cahn. ${ }^{1}$ Due to its quasiperiodicity, it was not possible to index the planes and axes of this phase in the usual way. To be able to index the reciprocal lattice spots as a combination of integers, at least six basis vectors are required for the icosahedral phase. Three systems for the indexing of icosahedral quasicrystals are in vogue. Elser ${ }^{2}$ and Bancel et $a{ }^{3}{ }^{3}$ used six vectors pointing to the vertices of an icosahedron as the basis vectors. The six vectors chosen by Elser ${ }^{2}$ can be obtained by a projection of a cube in six dimensions to three dimensions. The Bragg vector for each diffraction peak is expressed as a linear combination of basis vectors multiplied by integer indices, scaled by a factor called the quasilattice parameter. The observable reciprocal space spot nearest to the transmitted beam along a fivefold direction (vertex vector) is indexed as (100000). Bancel indices can be obtained from Elser's by a $\tau^{3}$ deflation. Cahn et $a l^{4}{ }^{4}$ chose a set of three basis vectors, similar to the cartesian coordinates, pointing to three of the fifteen twofold axes of the icosahedron. Indices that are irrational numbers related to the golden mean $\tau(=1+\sqrt{5} / 2)$ are expressed as a combination of two integers to obtain six integer indices in all. The three cartesian coordinates are expressed as six integer indices $\left(h / h^{\prime} k / k^{\prime} l / l^{\prime}\right)$ to separate out the integer and the irrational parts, so that the relationship between the indices and coordinates is $h / h^{\prime}=h+h^{\prime} \tau, k / k^{\prime}=k+k^{\prime} \tau, l / l^{\prime}=l+l^{\prime} \tau$.

The reciprocal space of the icosahedral quasicrystal has been studied by many workers. Chattopadhyay et al. ${ }^{5}$ and Singh and Ranganathan ${ }^{6}$ have used electron diffraction patterns for studying the reciprocal space. Dai and Wang ${ }^{7}$ have obtained and simulated the Holz lines of icosahedral quasicrystals. Programs are also available for the simulation of Kossel patterns of quasicrystals. ${ }^{8} \mathrm{Al}-$ though Kikuchi maps of the icosahedral phase and their indexing can readily be simulated, it is important to study the features and properties of this reciprocal space. Identifying important reciprocal vectors will help us know the appearance of these maps with increasing generation numbers in a simulation. A study of the indices of the important vectors and zone axes helps us understand the interrelationship between them and helps us know what kind of indices to expect for these planes and directions in three- and six-dimensional space. In the present work we bring out the important features of the icosahedral reciprocal space through identification and indexing of important reciprocal vectors and zone axes.

\section{ON THE INDEXING OF THE RECIPROCAL VECTORS AND THE ZONE AXES}

We will consider here the indexing schemes of Elser ${ }^{2}$ and Cahn et al. ${ }^{4}$ The Elser indices make use of the full symmetry of the icosahedral phase by considering it as a cube in six-dimensional space. On the other hand the planes and directions are more easily visualized using Cahn indices. Indices of Elser ${ }^{2}$ can be obtained from Cahn et al., ${ }^{4}$ and vice versa, by using relationships given by Cahn et al. ${ }^{4}$ However, these relationships as given by them are incorrect and we give below the corrected ones:

$n_{1}=\left(h+k^{\prime}\right) / 2, \quad n_{2}=\left(l+h^{\prime}\right) / 2, \quad n_{3}=\left(k+l^{\prime}\right) / 2$,

$n_{4}=\left(-h+k^{\prime}\right) / 2, \quad n_{5}=\left(k-l^{\prime}\right) / 2, \quad n_{6}=\left(-l+h^{\prime}\right) / 2$,

or, vice versa,

$$
\begin{aligned}
h & =n_{1}-n_{4} \quad h^{\prime}=n_{2}+n_{6} \\
k & =n_{3}+n_{5} \quad k^{\prime}=n_{1}+n_{4} \\
l & =n_{2}-n_{6} \quad l^{\prime}=n_{3}-n_{5} \quad .
\end{aligned}
$$


In addition to this, we derive some more relationships useful for indexing the icosahedral planes and directions. Because the Elser indexing scheme uses basis vectors that are orthogonal in the six-dimensional space, a zone law analogous to the cartesian system holds good. Thus if [abcdef] is a zone axis,

$$
n_{1} a+n_{2} b+n_{3} c+n_{4} d+n_{5} e+n_{6} f=0 .
$$

The Cahn indexing scheme uses an orthogonal set of three basis vectors in the three-dimensional space and therefore the zone law of the cartesian system can be extended to it. Thus the orthogonality condition between the reciprocal $\left(h / h^{\prime} k / k^{\prime} l / l^{\prime}\right)$ and the real space vector $\left(u / u^{\prime} v / v^{\prime} w / w^{\prime}\right)$ becomes

$$
\begin{aligned}
h u & +h^{\prime} u^{\prime}+k v+k^{\prime} v^{\prime}+l w+l^{\prime} w^{\prime} \\
& +\tau\left(h u^{\prime}+h^{\prime} u+h^{\prime} u^{\prime}+k v^{\prime}+k^{\prime} v+k^{\prime} v^{\prime}+l w^{\prime}\right. \\
& \left.+l^{\prime} w+l^{\prime} w^{\prime}\right)=0 .
\end{aligned}
$$

In this equation there are two type of terms: a set of integers and a set of irrational numbers. For this condition to hold, the sum of integers and the irrational numbers must separately be zero. This leads to a simple relation analogous to crystals and Eq. (3):

$$
h u+h^{\prime} u^{\prime}+k v+k^{\prime} v^{\prime}+l w+l^{\prime} w^{\prime}=0 .
$$

The zone axis between two reciprocal vectors $\left(h_{1} / h_{1}{ }^{\prime}\right.$ $\left.k_{1} / k_{1}{ }^{\prime} l_{1} / l_{1}{ }^{\prime}\right)$ and $\left(h_{2} / h_{2}{ }^{\prime} k_{2} / k_{2}{ }^{\prime} l_{2} / l_{2}{ }^{\prime}\right)$ is

$$
\begin{aligned}
& u=k_{1} l_{2}-k_{2} l_{1}+k_{1}^{\prime} l_{2}^{\prime}-k_{2}^{\prime} l_{1}^{\prime} \\
& u^{\prime}=k_{1} l_{2}^{\prime}-k_{2} l_{1}^{\prime}+k_{1}^{\prime} l_{2}-k_{2}^{\prime} l_{1}+k_{1}^{\prime} l_{2}^{\prime}-k_{2}^{\prime} l_{1}^{\prime} \\
& v=l_{1} h_{2}-l_{2} h_{1}+l_{1}^{\prime} h_{2}^{\prime}-l_{2}^{\prime} h_{1}^{\prime} \\
& v^{\prime}=l_{1}^{\prime} h_{2}-l_{2}^{\prime} h_{1}+l_{1} h_{2}^{\prime}-l_{2} h_{1}^{\prime}+l_{1}^{\prime} h_{2}^{\prime}-l_{2}^{\prime} h_{1}^{\prime} \\
& w=h_{1} k_{2}-h_{2} k_{1}+h_{1}^{\prime} k_{2}^{\prime}-h_{2}^{\prime} k_{1}^{\prime} \\
& w^{\prime}=h_{1}^{\prime} k_{2}-h_{2}^{\prime} k_{1}+h_{1} k_{2}^{\prime}-h_{2} k_{1}^{\prime}+h_{1}^{\prime} k_{2}^{\prime}-h_{2}^{\prime} k_{1}^{\prime} .
\end{aligned}
$$

Because the Cahn indexing system uses essentially the cartesian coordinate system, similar vectors or axes can have up to five kinds of indices related by a fivefold rotation matrix. For Cahn indices this matrix for the rotation axis $[1 / 00 / 10 / 0]$ is given as

$$
R=1 / 2\left(\begin{array}{rrrrrr}
1 & 0 & -1 & 1 & 0 & 1 \\
0 & 1 & 1 & 0 & 1 & 1 \\
-1 & 1 & 0 & 1 & -1 & 0 \\
1 & 0 & 1 & 1 & 0 & -1 \\
0 & -1 & 1 & 0 & -1 & 1 \\
-1 & -1 & 0 & 1 & 1 & 0
\end{array}\right) .
$$

Because a fivefold axis is the rotation axis itself, it has only one form of Cahn or cartesian indices. The twofold and threefold axes lie on vertices of the Moebius triangle and have two forms of indices each. The zone axes that lie on the sides of the Moebius triangle (i.e., the zone axes containing twofold vectors) have cartesian or Cahn indices in three forms. The axes that lie inside the triangle have five forms of indices.

\section{IMPORTANT FEATURES OF THE ICOSAHEDRAL PHASE RECIPROCAL SPACE}

To simulate the diffraction patterns of an icosahedral phase Chattopadhyay et al. ${ }^{5}$ used the Landau generation scheme as described by Nelson and Sachde. ${ }^{9}$ In this scheme, all the reciprocal vectors are sequentially generated by a combination of the vertex vectors of an icosahedron. As the generation number increases, the intensities of the reciprocal spots are expected to fall. Thus the most important reciprocal vectors, corresponding to most intense spots, will have the lowest generation numbers. Kikuchi lines/Holz lines corresponding to these low generations will be observed most intensly. Let us now point out which are these vectors.

\section{A. Important vectors}

Table I lists all possible vectors up to the sixth generation. Each type of vector is given a name V1, V2, etc., and its length is given with respect to the vertex vector V1 whose length is taken as unity. There are 12 firstgeneration vectors (V1), corresponding to the fivefold directions. There are 60 different combinations of two vertex vectors. These 60 combinations generate only 30 second-generation vectors, the twofold vectors (V2), because each twofold direction can result from two different combinations of two-vertex vectors. For example, the vectors (110000) and (001001) point in the same twofold symmetry direction. The magnitude of the vectors obtained by these two different combinations is scaled by $\tau$.

There are 160 different combinations of three vertex vectors. The third-generation vectors are V3 (threefold vector), V4, and V5. Each of the threefold directions can also be obtained by the addition of two different combinations of three vertex vectors. The magnitudes of the vectors obtained by these two different combinations are scaled by $\tau^{3}$. Thus 40 combinations of three-vertex vectors are accounted for by the 20 threefold vectors. There are 60 each of V4 and V5 vectors.

There are 240 different combinations of four vertex vectors in an icosahedron. Thirty of these combinations result in twofold vectors scaled by $\tau$ and another 30 also in the twofold directions scaled by $1 / \tau^{2}$. Thus 60 of these combinations result in vectors along the twofold directions. Sixty combinations generate the V6 vector while another 60 generate vectors in the same direction with 
TABLE I. The generation of vectors from the basis vectors.

\begin{tabular}{|c|c|c|c|c|c|}
\hline Generation number & Type & Example & Magnitude of vector, $|g|$ & Vector (direction) & Number of vectors \\
\hline First & 1 & 100000 & 1.0000 & $\mathrm{~V} 1(\mathrm{~K})$ & 12 \\
\hline \multirow[t]{2}{*}{ Second } & 1 & 110000 & 1.7013 & V2 (A) & 30 \\
\hline & 2 & 001001 & 1.0515 & $\tau^{-1} \mathrm{~V} 2(\mathrm{~A})$ & 30 \\
\hline \multirow[t]{4}{*}{ Third } & 1 & 111000 & 2.3840 & V3 (D) & 20 \\
\hline & 2 & $0001 \overline{1} 1$ & 0.5628 & $\tau^{-3} \mathrm{~V} 3(\mathrm{D})$ & 20 \\
\hline & 3 & $11000 \overline{1}$ & 1.4511 & V4 (G) & 60 \\
\hline & 4 & 110100 & 1.9734 & V5 (O) & 60 \\
\hline \multirow[t]{5}{*}{ Fourth } & 1 & 111001 & 2.7528 & $\tau \mathrm{V} 2(\mathrm{~A})$ & 30 \\
\hline & 2 & $11 \overline{1} 00 \overline{1}$ & 0.6498 & $\tau^{-2} \mathrm{~V} 2(\mathrm{~A})$ & 30 \\
\hline & 3 & 111010 & 2.4060 & V6 (I) & 60 \\
\hline & 4 & $1001 \overline{1} 1$ & 1.4870 & $\tau^{-1} \mathrm{~V} 6(\mathrm{I})$ & 60 \\
\hline & 5 & $11010 \overline{1}$ & 2.0000 & (F) & 60 \\
\hline \multirow[t]{4}{*}{ Fifth } & 1 & 011111 & 2.2361 & $\left(\tau^{2}+1\right) / \tau \mathrm{V} 1(\mathrm{~K})$ & 12 \\
\hline & 2 & $11101 \overline{1}$ & 2.2361 & $\ldots$ & 60 \\
\hline & 3 & 111110 & 2.9288 & $\ldots$ & 60 \\
\hline & 4 & $110 \overline{1} 1 \overline{1}$ & 1.1926 & $\ldots$ & 60 \\
\hline \multirow[t]{4}{*}{ Sixth } & 1 & $\overline{1} 11111$ & 1.2361 & $2 \tau^{-1} \mathrm{~V} 1(\mathrm{~K})$ & 12 \\
\hline & 2 & 111111 & 3.2361 & $2 \tau \mathrm{V} 1(\mathrm{~K})$ & 12 \\
\hline & 3 & $111 \overline{1} 1 \overline{1}$ & 1.8212 & $2 \tau^{-2} \mathrm{~V} 3(\mathrm{D})$ & 20 \\
\hline & 4 & $1111 \overline{1} 1$ & 2.9467 & $2 \tau^{-1} \mathrm{~V} 3(\mathrm{D})$ & 20 \\
\hline
\end{tabular}

lengths scaled by $1 / \tau$. The rest of the 60 combinations result in $60 \mathrm{~F}$ directions which will be pointed out on the stereogram.

There are 192 different combinations of five vertex vectors. Twelve of these produce vectors along vertex vectors with lengths $\left(\tau^{2}+1\right) / \tau$. The rest of the combinations result in three kinds of vectors unidentified in this study. That there are 60 of each kind of vector indicates that these vectors, too, lie on the twofold vector traces. In cartesian coordinates, these vectors are of the kind [ 10 $\left.\tau^{5}\right],\left[\begin{array}{llll}\tau^{2}+1 & 0 & \tau^{3}\end{array}\right]$ and $\left[\begin{array}{lll}1 & 0 & \tau^{3}+\tau\end{array}\right]$.

There are 64 possible combinations of six-vertex vectors. Twenty-four of these result in vectors of two different lengths along the fivefold directions, while 40 others generate vectors of two different lengths along threefold directions.

We will now draw these vectors on a stereogram. Singh and Ranganathan ${ }^{6}$ drew the stereogram of an icosahedral phase with great circles corresponding to four reciprocal vectors, which are the vertex vector $\mathrm{V} 1$, the edge vector V2, the face vector V3, and the "fourth" vector, which is V4. These four vectors are also observed in the Kikuchi bands of Al-Mn icosahedral phase mapped by Field. ${ }^{10}$ Figure 1(a) is drawn from their experimental pattern. This triangle ADK (A is twofold, D is threefold, and $\mathrm{K}$ is fivefold axis) is bounded by twofold $\mathrm{V} 2$ bands. Bands corresponding to vector V1 make zone axes at $\mathrm{A}$ and $\mathrm{F}$. Bands corresponding to $\mathrm{V} 3$ make zone axes at A, I, and M. V4 make zones at A, H, P, F, B, I, $\mathrm{L}$, and $\mathrm{J}$. In addition, there is one more prominent band, which is the V6 vector. This band passes through A, G, $\mathrm{Q}, \mathrm{D}, \mathrm{O}$, and $\mathrm{I}$. This vector has been shown to be a fourth-generation vector.
An experimental determination and computersimulated Holz line pattern connected from seven patterns covering the whole orientation triangle of the primitive Al-Mn-Si icosahedral phase has been shown by Dai and Wang, ${ }^{7}$ reproduced in Fig. 1(b). It exhibits the prominent reciprocal vectors V1, V2, and V3. The V4 vector is not identified. Instead, the V5 vector is observed here which is present in the A pattern and crosses the twofold vector trace at E. Just as in the V4 vector, there are seven variants of this vector present in the Moebius triangle. Two each of these variants are present in the B, $\mathrm{F}, \mathrm{I}$, and $\mathrm{P}$ zone axes, just as in the $\mathrm{V} 4$ vector.

Figure 1(c) shows a Moebius triangle of an icosahedral phase stereogram in which traces of all the six vectors V1-V6 are drawn. The major zone axes A, D, F, and K are labeled with indices of Cahn et al. ${ }^{4}$ Table II shows the indices of reciprocal vector traces present in the Moebius triangle of Fig. 1(c) in Cahn et al. ${ }^{4}$ and Elser ${ }^{2}$ notations. These indices are for a primitive icosahedral phase. In case of an ordered face-centered icosahedral phase these indices are doubled due to a doubling of the quasilattice parameter. There is one trace of the vector of type V1, three of type V2, two of type V3, and seven of type V4 and V5 and five of the kind V6 in this stereographic triangle. There is a threefold vector of the kind (111) (or $\left(\begin{array}{lll}1 / 1 & 1 / 1 & 1 / 1\end{array}\right)$, which corresponds to a threefold rotation axis in a cube. That the V1 vector is a first-generation vector, V2 is second generation, V3, V4, and V5 are third-generation, and V6 is a fourth-generation vector is seen from the addition of the integers in the Elser indices.

The number of variants of each type of vector that will exist in a unit stereographic triangle will be half the number of such triangles it crosses between two twofold 


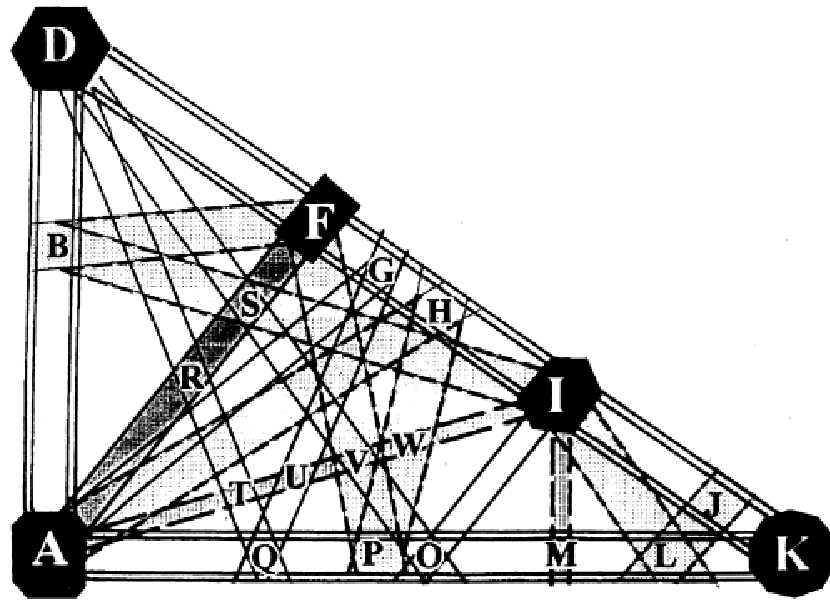

(a)

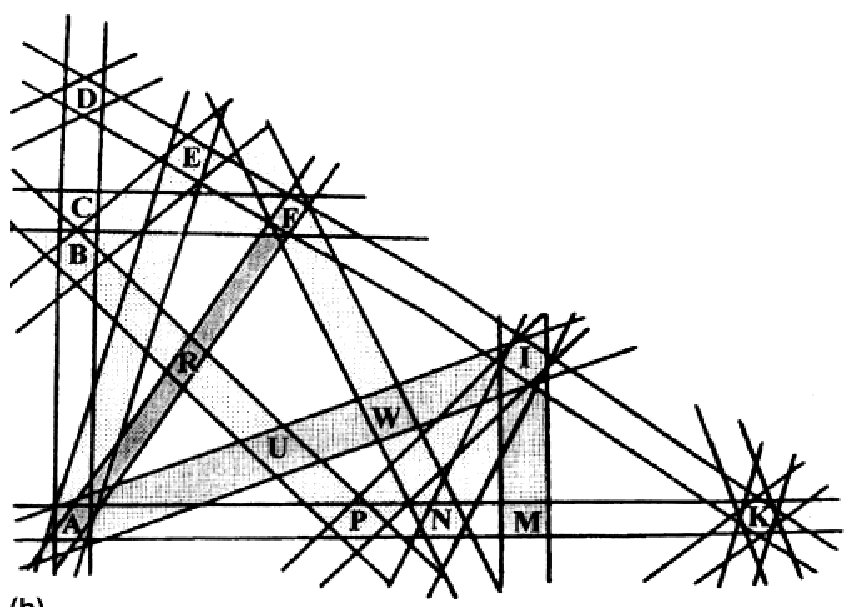

(b)

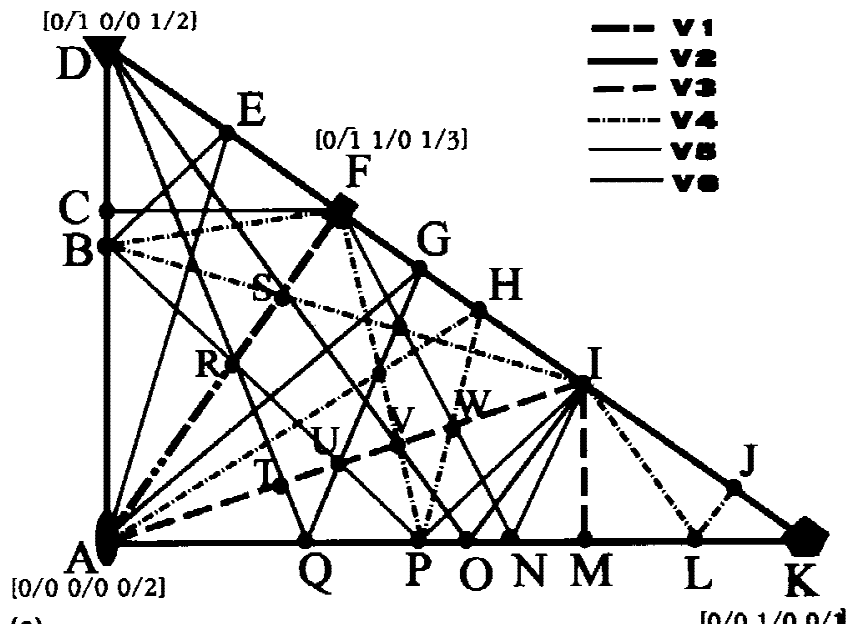

(c)

FIG. 1. (a) Kikuchi map of an Al-Mn icosahedral phase. ${ }^{10}$ (b) Holz lines map of an Al-Mn-Si icosahedral phase. ${ }^{7}$ (c) An icosahedral phase stereogram showing traces of six types of vectors V1-V6 making zone axes marked $\mathrm{A}$ to $\mathrm{W}$. Indices are assigned to four prominent zone axes. Indices of all the traces are listed in Table II. zone axes. For example, a V1 trace intersects only two unit triangles between two twofold A zone axes. A V3 trace crosses four unit triangles between two successive "A" zone axes. There are two traces of the V3 kind in each triangle. The V4 and V5 vectors pass 14 triangles. Seven traces of each kind are present in each triangle. Only five traces of V6 vectors are observed in a triangle because each of these traces pass through only ten basic triangles, instead of 14 like V4 and V5. This is because these traces also pass through the $\mathrm{D}$ axis, which forms a corner of the unit triangle, just as A.

Vectors V2 and V6 are even parity vectors and thus follow a $\tau$ inflation rule for the reciprocal spots along them. The vectors V1, V3, V4, and V5 are odd parity vectors. They follow a $\tau^{3}$ inflation rule in case of the primitive icosahedral phase and $\tau$ inflation in case of the face-centered ordered icosahedral phase. Thus the reciprocal spots along these vectors are spaced out in the primitive phase.

\section{B. The zone axes}

As observed in Fig. 1(c), the twofold zone axis A contains traces of all the six kinds of vectors. The zone axis I contains traces of all vectors except V1. The zone F contains vectors V1, V2, V4, and V5. Both the zones B and $\mathrm{P}$ contain V2, V4, and V5 and look identical. The zone D (threefold) contains three variants of V2 and six of V6. The fivefold zone $\mathrm{K}$ is made of five variants of V2. The zone axes E, G, H, L, N, O, and Q consist of one variant of $\mathrm{V} 2$ and two of either V4, V5, or V6 vectors. The zone axes $\mathrm{C}$, J, and $\mathrm{M}$ have V5, V4, and $\mathrm{V} 3$ vectors, respectively, at right angles to one of the variants of $\mathrm{V} 2$.

In addition to the zone axes identified above, there are a number of intersections inside the triangle. The zones made by all of these intersections may not be very important. However, V1 being the most prominent vector, the zone axes made by it should be important. In addition, the zone axes made by V3 are also considered here. The vector V1 makes a zone axis $\mathrm{R}$ in which one variant each of V5 and V6 are present. Another zone axis S is constituted by one variant each of V1, V4, and V6. These two zone axes are identified by Field ${ }^{10}$ as well. The zone axes made by $\mathrm{V} 3$ are $\mathrm{T}, \mathrm{U}, \mathrm{V}$, and $\mathrm{W}$. A V6 vector trace crosses $\mathrm{V} 3$ at $90^{\circ}$ at $\mathrm{T}$. The zone $\mathrm{U}$ is formed by one trace each of V3, V5, and V6; zone V by V3, V4 and V6; and zone $\mathrm{W}$ by $\mathrm{V} 3, \mathrm{~V} 4$, and V5.

Table III lists one type of index for the zone axes A to $\mathrm{Q}$ in the cartesian system and the corresponding Elser indices. Cartesian coordinates are given for easy comparison among other indices. These can readily be converted to Cahn indices and other equivalent indices can be generated by using the matrix 7 . Determination of the zone axis indices shows that the poles for the V4 vector 
TABLE II. Indices of the reciprocal vector traces shown in the stereographic section of the icosahedral phase in Fig. 1(c).

\begin{tabular}{|c|c|c|c|c|c|c|c|c|c|c|c|c|}
\hline \multirow{3}{*}{$\frac{\text { Vector }}{\text { V1 }}$} & \multicolumn{12}{|c|}{ Indices } \\
\hline & \multicolumn{3}{|c|}{ Cartesian } & \multicolumn{3}{|c|}{ Cahn } & \multicolumn{6}{|c|}{ Elser } \\
\hline & $\overline{1}$ & $\bar{\tau}$ & 0 & $\overline{1} / 0$ & $0 / \overline{1}$ & $0 / 0$ & $\overline{1}$ & 0 & 0 & 0 & 0 & 0 \\
\hline $\mathrm{V} 2$ & 0 & 1 & 0 & $0 / 0$ & $0 / 2$ & $0 / 0$ & 1 & 0 & 0 & 1 & 0 & 0 \\
\hline $\mathrm{V} 2$ & $\overline{1}$ & 0 & 0 & $0 / \overline{2}$ & $0 / 0$ & $0 / 0$ & 0 & $\overline{1}$ & 0 & 0 & 0 & $\overline{1}$ \\
\hline $\mathrm{V} 2$ & $\tau^{2}$ & $\bar{\tau}$ & 1 & $1 / 1$ & $0 / \overline{1}$ & $1 / 0$ & 0 & 1 & 0 & $\overline{1}$ & 0 & 0 \\
\hline V3 & $\tau^{2}$ & 1 & 0 & $1 / 2$ & $0 / 1$ & $0 / 0$ & 1 & 1 & 0 & 0 & 0 & 1 \\
\hline V3 & 0 & $\bar{\tau}^{2}$ & 1 & $0 / 0$ & $\overline{1} / \overline{2}$ & $0 / 1$ & $\overline{1}$ & 0 & 0 & $\overline{1}$ & $\overline{1}$ & 0 \\
\hline V4 & $\tau^{2}+1$ & $\tau^{2}$ & 0 & $\overline{1} / 2$ & $0 / 1$ & $0 / 0$ & 0 & 1 & 0 & 1 & 0 & 1 \\
\hline V4 & $\overline{1}$ & $\bar{\tau}^{3}$ & 1 & $1 / \overline{1}$ & $\overline{1} / \overline{1}$ & $\overline{1} / 1$ & 0 & $\overline{1}$ & 0 & $\overline{1}$ & $\overline{1}$ & 0 \\
\hline V4 & 1 & $\bar{\tau}^{3}$ & 1 & $\overline{1} / 1$ & $\overline{1} / \overline{1}$ & $\overline{1} / 1$ & $\overline{1}$ & 0 & 0 & 0 & $\overline{1}$ & 1 \\
\hline V4 & $\tau^{3}$ & 1 & 1 & $1 / 1$ & $\overline{1} / 1$ & $\overline{1} / 1$ & 1 & 0 & 0 & 0 & $\overline{1}$ & 1 \\
\hline V4 & $\tau^{3}$ & $\overline{1}$ & 1 & $1 / 1$ & $1 / \overline{1}$ & $\overline{1} / 1$ & 0 & 0 & 1 & $\overline{1}$ & 0 & 1 \\
\hline V4 & $\tau$ & $\overline{2}$ & 1 & $0 / 1$ & $\overline{2} / 0$ & $1 / 0$ & 0 & 1 & $\overline{1}$ & 0 & $\overline{1}$ & 0 \\
\hline V4 & $\bar{\tau}$ & $\overline{2}$ & 1 & $0 / \overline{1}$ & $\overline{2} / 0$ & $1 / 0$ & 0 & 0 & $\overline{1}$ & 0 & $\overline{1}$ & $\overline{1}$ \\
\hline V5 & $\overline{1}$ & $\tau^{2}+1$ & 0 & $\overline{1} / 0$ & $2 / 1$ & $0 / 0$ & 0 & 0 & 1 & 1 & 1 & 0 \\
\hline V5 & $\tau^{3}$ & $\tau^{3}$ & 1 & $1 / 1$ & $1 / 1$ & $\overline{1} / 1$ & 1 & 0 & 1 & 0 & 0 & 1 \\
\hline V5 & $\tau^{3}$ & $\bar{\tau}^{3}$ & $\overline{1}$ & $1 / 1$ & $\overline{1} / \overline{1}$ & $1 / \overline{1}$ & 0 & 1 & $\overline{1}$ & $\overline{1}$ & 0 & 0 \\
\hline V5 & $\bar{\tau}^{3}$ & $\bar{\tau}^{3}$ & 1 & $\overline{1} / \overline{1}$ & $\overline{1} / \overline{1}$ & $\overline{1} / 1$ & $\overline{1}$ & $\overline{1}$ & 0 & 0 & $\overline{1}$ & 0 \\
\hline V5 & $\bar{\tau}$ & $2 \bar{\tau}$ & 1 & $0 / \overline{1}$ & $0 / \overline{2}$ & $1 / 0$ & $\overline{1}$ & 0 & 0 & $\overline{1}$ & 0 & $\overline{1}$ \\
\hline V5 & $\tau$ & $2 \bar{\tau}$ & 1 & $0 / 1$ & $0 / \overline{2}$ & $1 / 0$ & $\overline{1}$ & 1 & 0 & $\overline{1}$ & 0 & 0 \\
\hline V5 & $\tau^{2}+1$ & 0 & 1 & $2 / 1$ & $0 / 0$ & $1 / 0$ & 1 & 1 & 0 & $\overline{1}$ & 0 & 0 \\
\hline V6 & $\overline{1}$ & $\overline{1}$ & 0 & $0 / \overline{1}$ & $0 / \overline{1}$ & $0 / 0$ & $\overline{1}$ & $\overline{1}$ & 0 & $\overline{1}$ & 0 & $\overline{1}$ \\
\hline V6 & $\bar{\tau}^{2}$ & $\bar{\tau}^{4}$ & 1 & $0 / \overline{1}$ & $\overline{1} / \overline{2}$ & $\overline{1} / 1$ & $\overline{1}$ & $\overline{1}$ & 0 & $\overline{1}$ & $\overline{1}$ & 0 \\
\hline V6 & $\tau^{2}$ & $\bar{\tau}^{4}$ & 1 & $0 / 1$ & $\overline{1} / \overline{2}$ & $\overline{1} / 1$ & $\overline{1}$ & 0 & 0 & $\overline{1}$ & $\overline{1}$ & 1 \\
\hline V6 & $\tau^{2}$ & $\bar{\tau}^{2}+\overline{1}$ & 1 & $1 / 1$ & $\overline{2} / \overline{1}$ & $1 / 0$ & 0 & 1 & $\overline{1}$ & $\overline{1}$ & $\overline{1}$ & 0 \\
\hline V6 & $\bar{\tau}^{2}$ & $\bar{\tau}^{2}+\overline{1}$ & 1 & $\overline{1} / \overline{1}$ & $\overline{2} / \overline{1}$ & $1 / 0$ & $\overline{1}$ & 0 & $\overline{1}$ & 0 & $\overline{1}$ & $\overline{1}$ \\
\hline
\end{tabular}

traces are $\mathrm{G}$ zone axes, for $\mathrm{V} 5$ vector traces are $\mathrm{O}$ zone axes, and for V6 vectors are I zone axes. Because these axes fall on the sides of the basic triangle, the cartesian and Cahn indices of these vectors also assume three forms and there are 30 axes of each of these kinds in an icosahedron.

Of the indices of zone axes on the trace of the vector (010) listed in Table III, midway between the two twofold zone axes A [001] and [100] lies the axis I [101]. The mirrorlike nature of this axis has been discussed earlier by Chattopadhyay et al. ${ }^{5}$ This axis corresponds to a cubic twofold axis. On either side of this axis I are axes with indices permuted $[\mathrm{u} 0 \mathrm{w}]$ to $[\mathrm{w} 0 \mathrm{u}]$. However, these axes are not equivalent in the icosahedral symmetry because the indices can be cyclically rotated but not permuted to find equivalent ones. This can be checked by the matrix 7. This inequivalence is brought out by very different Elser indices of these axes. Axes A, E, I, and L coincide with cubic axes.

The zone axes that are inside the stereographic triangle

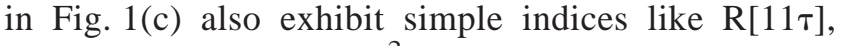
$\mathrm{S}[\tau 1 \tau], \mathrm{T}[112]$, and $\mathrm{U}\left[11 \tau^{2}\right]$, bringing out the importance of these axes. The axis T[112] coincides with a cubic axis. The indices of the zone axes $\mathrm{R}$ and $\mathrm{S}$ made by $\mathrm{V} 1$

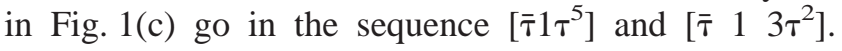
Similarly, the indices of the zone axes T, U, V, and W, made by V3, in Fig. 1(c), follow a trend $\left[1 \tau^{2} \tau^{6}+\tau^{2}\right]$, $\left[\overline{1} \tau^{2} \tau^{5}+\tau^{3}\right],\left[\overline{1} \tau^{2} \tau^{5}+1\right]$ and $\left[\overline{1} \tau^{2} \tau^{4}+2 \tau\right]$.
The cubic coordinates make working with the stereogram much simpler. However, each term involving the golden mean $\tau$ can be written in many different ways, which may not be recognized to be the same. This ambiguity is removed in Cahn indices by separating out the rational and irrational parts of an index. Furthermore, the Cahn system uses only integers that are easy to use, as demonstrated in Sec. II.

\section{CONCLUSIONS}

The reciprocal space of the icosahedral quasicrystal has been studied to identify its important features and the following conclusions are made.

(1) Reciprocal vectors generated by icosahedral vertex vectors up to six generations are explored. The six most prominent of these (V1-V6) are recognized in the available Kikuchi and Holz line maps.

(2) Apart from the first-generation vectors, which are fivefold, and the second generation, which are all twofold, there are three kinds of third-generation vectors, only one kind being threefold. There are two new kinds of vectors in the fourth generation but no new kinds of vectors in the sixth generation.

(3) The types of indices for all these vectors and zone axes $\mathrm{A}-\mathrm{W}$ are determined. In order to index, some more relationships for indexation are derived. It is shown that in the Cahn system of indexing too the zone law is analogous to that valid for crystals. 
(4) The pole of the vector V1 (fivefold) is at K, V2 (twofold) is at A, V3 (threefold) is at D, V4 (third generation) is at G, V5 (third generation) is at O, and V6 (fourth generation) is at I. In addition, at $\mathrm{F}$ is the pole of another fourth-generation vector.

TABLE III. Cartesian indices of zone axes A to Q, which lie on the trace of the twofold reciprocal vector (010), to bring out the relationship between the various axes.

\begin{tabular}{|c|c|c|c|c|c|c|c|c|c|}
\hline \multirow{3}{*}{$\frac{\text { Label }}{\text { A (twofold) }}$} & \multicolumn{9}{|c|}{ Indices } \\
\hline & \multicolumn{3}{|c|}{ Cartesian } & \multicolumn{6}{|c|}{ Elser } \\
\hline & 0 & 0 & 1 & 0 & 0 & 1 & 0 & $\overline{1}$ & 0 \\
\hline $\mathrm{B}$ & 1 & 0 & $\tau^{3}$ & $\overline{1}$ & 2 & 1 & 1 & $\overline{1}$ & 0 \\
\hline $\mathrm{C}$ & 1 & 0 & $\tau^{2}+1$ & $\overline{1}$ & 3 & 2 & 1 & $\overline{2}$ & 1 \\
\hline D (threefold) & 1 & 0 & $\tau^{2}$ & 0 & 1 & 1 & 0 & $\overline{1}$ & 0 \\
\hline $\mathrm{E}$ & 1 & 0 & 2 & 0 & 1 & 2 & 0 & $\overline{2}$ & 1 \\
\hline $\mathrm{F}$ & 1 & 0 & $\tau$ & 1 & 0 & 1 & $\overline{1}$ & $\overline{1}$ & 0 \\
\hline G & $\tau^{2}$ & 0 & $\tau^{2}+1$ & 0 & 0 & 1 & 0 & $\overline{1}$ & 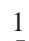 \\
\hline $\mathrm{H}$ & $\tau$ & 0 & 2 & 0 & 3 & 0 & 0 & 0 & \\
\hline I & 1 & 0 & 1 & 0 & 1 & 1 & 0 & $\overline{1}$ & 1 \\
\hline $\mathrm{J}$ & $\tau^{2}+1$ & 0 & $\tau^{2}$ & 2 & 2 & 1 & $\overline{2}$ & $\overline{1}$ & 0 \\
\hline K (fivefold) & $\tau$ & 0 & 1 & 0 & 1 & 0 & 0 & 0 & 0 \\
\hline $\mathrm{L}$ & 2 & 0 & 1 & 0 & 2 & 1 & 0 & $\overline{1}$ & 2 \\
\hline M & $\tau^{2}$ & 0 & 1 & 1 & 2 & 0 & $\overline{1}$ & 0 & 0 \\
\hline $\mathrm{N}$ & $2 \tau$ & 0 & 1 & 0 & 3 & 0 & 0 & 0 & 1 \\
\hline $\mathrm{O}$ & $\tau^{2}+1$ & 0 & 1 & 1 & 1 & 0 & $\overline{1}$ & 0 & 0 \\
\hline $\mathrm{P}$ & $\tau^{3}$ & 0 & 1 & 1 & 0 & 1 & $\overline{1}$ & $\overline{1}$ & 2 \\
\hline Q & $\tau^{4}$ & 0 & 1 & 0 & $\overline{1}$ & 1 & 0 & $\overline{1}$ & 2 \\
\hline
\end{tabular}

\section{ACKNOWLEDGMENTS}

The authors are grateful to Professor K. Chattopadhyay, Dr. B.B. Rath, Dr. C.S. Pande, Professor S. Mahajan, Dr. U. Dahmen, and Mr. A.K. Srivastava for stimulating discussions. Financial support from the Office of Naval Research, U.S.A., under the Indo-U.S. cooperation project entitled "Quasicrystals and Crystalline Interfaces" (ONR Grant N 00014-95-1-0095) is gratefully acknowledged.

\section{REFERENCES}

1. D. Shechtman, I. Blech, D. Gratias, and J.W. Cahn, Phys. Rev. Lett. 53, 1951 (1984).

2. V. Elser, Phys. Rev. B 32, 4892 (1985).

3. P.A. Bancel, P.A. Heiney, P.W. Stephens, A.I. Goldman, and P.M. Horn, Phys. Rev. Lett. 54, 2422 (1985).

4. J.W. Cahn, D. Shechtman, and D. Gratias, J. Mater. Res. 1, 13 (1986).

5. K. Chattopadhyay, S. Lele, R. Prasad, S. Ranganathan, G.N. Subbanna, and N. Thangaraj, Scripta Metall. 19, 1331 (1985).

6. A. Singh and S. Ranganathan, Scripta Metall. Mater. 25, 409 (1991).

7. M. Dai and R. Wang, Solid State Comm. 73, 77 (1990).

8. S. Weber, J. Appl. Cryst. 30, 85 (1997).

9. D.R. Nelson and S. Sachdev, Phys. Rev. B: Solid State Condens. Mater. 32, 689 (1985).

10. R.D. Field (unpublished) 